\title{
Left Para-Renal Castleman Disease: Case Report
}

\author{
Flavius Mocian, Sorin Sorlea, Marius Coros \\ Department of Surgery, Clinical County Hospital Mureș, Tîrgu Mureș, Romania
}

\section{CORRESPONDENCE}

\section{Flavius Mocian}

Str. Gheorghe Marinescu nr. 1

540103 Tîrgu Mureș, Romania

E-mail:mocianfla@gmail.com

\section{ARTICLE HISTORY}

Received: April 14, 2018

Accepted: May 28, 2018
Tel. +40770842781

\begin{abstract}
Castleman disease represents a rare lymphoproliferative disorder of unknown etiology. It is usually located in the mediastinum and in very few cases in the retroperitoneal space. We present the case of a 43-year-old male patient with a retroperitoneal tumor that was incidentally diagnosed during an abdominal computed tomography scan. The patient underwent surgery by open approach, and the tumor, which was adherent to the superior pole of the left kidney, was entirely removed. The histology examination revealed a vascular-hyalinetype Castleman disease. The postoperative evolution was uneventful, with no signs of tumor recurrence at the 4-month check-up. The surgeon should be aware of the possible retroperitoneal location of Castleman disease, even if it is a rare occurrence, and a complete removal of the tumor is followed by a favorable long-term prognosis.
\end{abstract}

Keywords: Castleman disease, hyaline vascular type, retroperitoneum, pararenal

\section{INTRODUCTION}

Angiofollicular hyperplasia, also known as Castleman disease, represents a rare lymphoproliferative disorder with unknown etiology, which usually develops in the mediastinum ( $70 \%$ of cases), in $7 \%$ of cases in the retroperitoneum, and only in $2 \%$ of patients in the pararenal space. ${ }^{1}$

\section{CASE REPORT}

A 43-year-old patient was admitted to the Department of Occupational Medicine for osteoarticular pains of professional etiology (physical overload). Among other investigations, an abdominal and pelvic computed tomography (CT) scan with oral and intravenous contrast agent was performed. The examination highlighted a solid, iodophilic tumor with microcalcifications located posteriorly to the pancreas tail, adjacent to the upper pole of the left kidney, with well-defined edges and a cleavage plane (Figure 1). The vertical extension of the tumor was of $67 \mathrm{~mm}$, with the maximum axial diameters of $64 / 51 \mathrm{~mm}$. The patient was referred to the surgical department. 


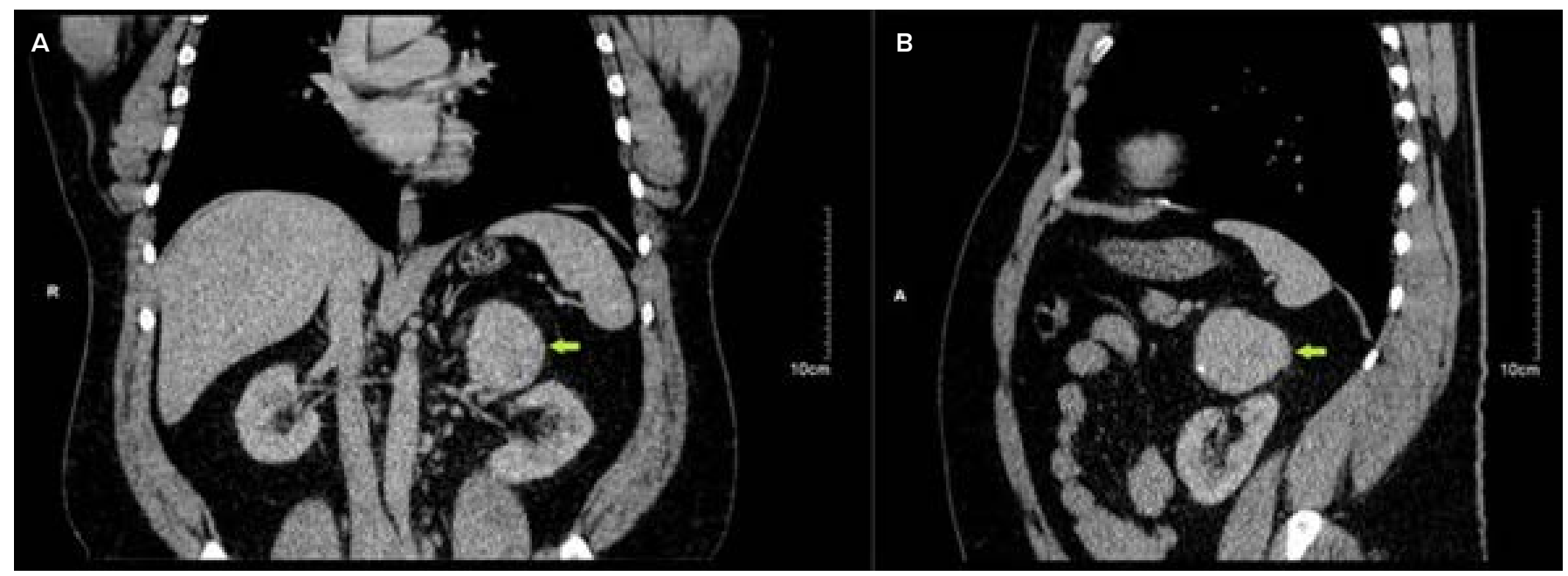

FIGURE 1. CT imaging showing a well-defined retroperitoneal tumor adjacent to the upper pole of the left kidney. A - Coronal reconstruction (arrow); B - Axial reconstruction (arrow).

The patient underwent surgery by a classical open approach. The intraoperative findings were that of a well-defined, mobile tumor, of soft consistency, located between the upper left renal pole, the spleen, and the posterior aspect of the stomach. A complete removal of the tumor was carried out. The postoperative evolution was uneventful, with complete recovery and discharge on the 9th day after surgery.

The anatomical gross aspect of the tumor on sections was that of a well-defined, $6 \mathrm{~cm}$ diameter node, with a whitish aspect, of elastic consistency, not exceeding the edges of the excision.

The histopathological findings were that of a lymph node with unaltered follicular structure; follicles with diminished germinal centers penetrated by vascular structures and some hyalinization (Figure 2). In the interfollicular space, there was an expansion of the mantle area and capillary venous hyperplasia associated with hyalinization. The immunohistochemical profile was the following: CD20 and BCL-2 normal follicles, interfollicular T/

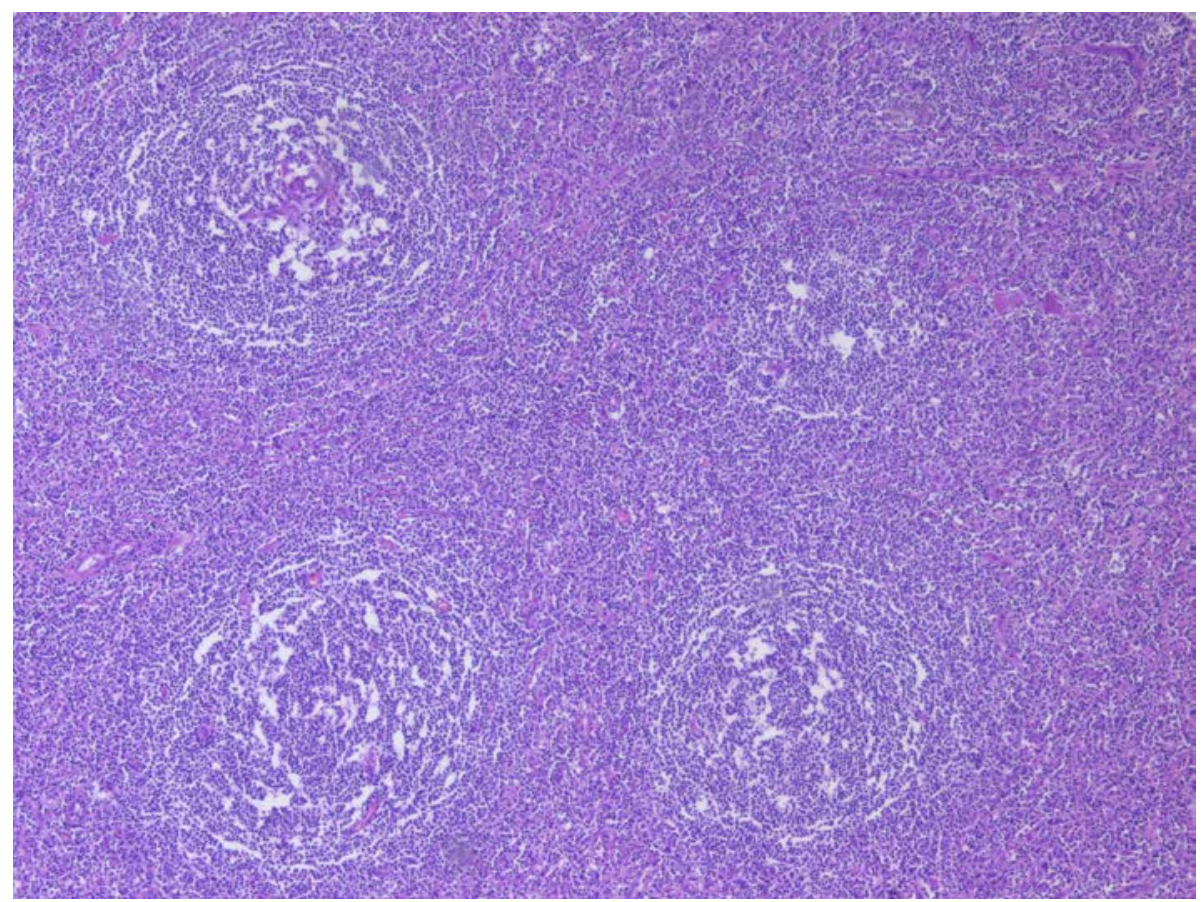

FIGURE 2. Histopathological aspect, Hematoxylin-Eosin, 40x 


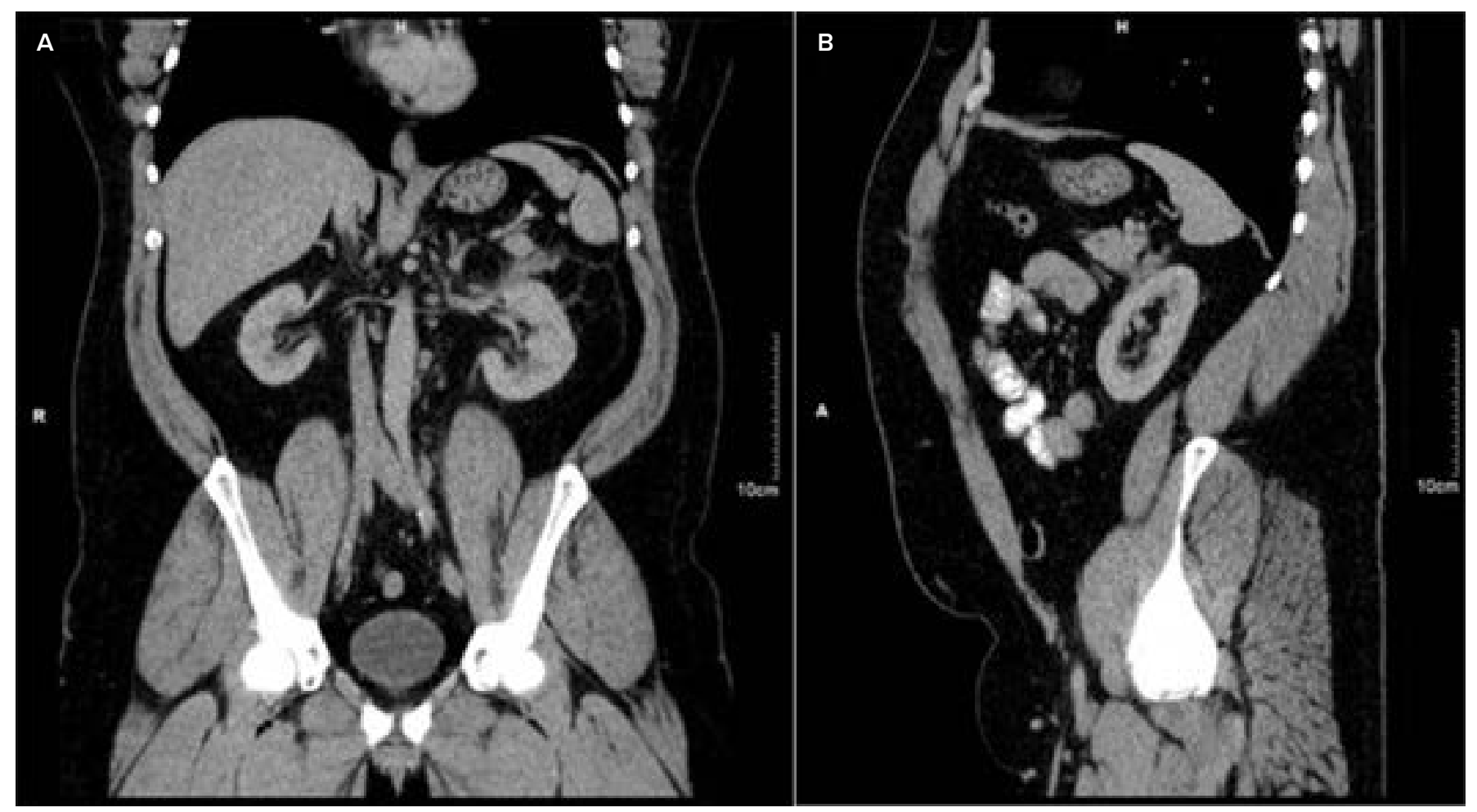

FIGURE 3. CT scan showing the postoperative aspect at the four-month follow-up. A - Coronal reconstruction; B - Sagittal view.

CD4 and CD8 positive lymphocytes, few B lymphocytes and plasma cells. There was no restriction of light kappa and light lambda chains.

Based on these examinations, the final diagnosis of vascular-hyaline-type Castleman disease was established.

At the four-month follow-up, the CT scan did not find any signs of tumor recurrence (Figure 3).

The patient agreed to the publication of his data, and the manuscript was written according with the ethical principles stated in the Declaration of Helsinki.

\section{DISCUSSIONS}

Castleman disease was first described by Benjamin Castleman in $1956 .{ }^{2}$ The most frequent location (about $70 \%$ of cases) reported by authors was the mediastinum and, in much more rare cases, the retroperitoneum (7\%) and the pararenal space (2\%). ${ }^{1,3}$ The case presented herein falls into the more rare group of cases with pararenal location.

From a clinical point of view, Castleman disease can be classified into a unicentric or multicentric disease, depending on the number of affected lymph nodes. ${ }^{1}$ Three histopathological types have been described: vascular-hyaline, plasma-cell, and mixed forms. ${ }^{4}$ In the presented case, there was a unicentric, retroperitoneal, vascular-hyaline-type tumor.
In $90 \%$ of cases, the vascular-hyaline type is unicentric, represented by an asymptomatic tumor with a benign evolution. ${ }^{5}$ In some cases, depending on the size of the tumor and its relationship to the adjacent organs, various symptoms may arise, from abdominal or lumbar pain to vomiting and weight loss. ${ }^{6}$ In our case, there were no tumor-related symptoms, the tumor being incidentally discovered by a CT scan, performed in a patient with joint complaints.

The treatment of Castleman disease, whether vascularhyaline or plasma-cell, consists in surgical resection of the entire tumor, with a favorable long-term prognosis. ${ }^{7,8} \mathrm{De}-$ pending on tumor size and location, the laparoscopic approach should be considered with a faster recovery of the patient compared to the open, classical approach. ${ }^{9}$

Radiotherapy is an alternative treatment, with a response rate up to $72 \%$ in cases where surgery is not applicable. $^{7}$

The long-term prognosis for unicentric Castleman disease after complete resection of the tumor is very good, with $95 \%$ survival in 10 years. ${ }^{10}$

\section{CONCLUSION}

Although in rare cases, retroperitoneal tumors may be represented by Castleman disease. The surgeon should con- 
sider this possibility because the complete removal of the tumor is followed by a very good long-term prognosis.

\section{CONFLICT OF INTEREST}

The authors declare no conflict of interests.

\section{REFERENCES}

1. Hatano K, Fujita S, Tsujimoto Y, et al. Rare case of the hyaline vascular type of Castlemans's disease of the kidney. International Journal of Urology. 2007:14:1098-1100.

2. Castleman B, Iverson L, Menendez VP. Localized mediastinal lymph-node hyperplasia resembling thymoma. Cancer. 1956;9:822-830.

3. Guthrie PJ, Thomas JV, Peker D, Turkbey B, Rais-Bahrami S. Perivesical unicentric Castleman disease initially suspected to be metastatic prostate cancer. Urol Ann. 2016;8:245-248.

4. Vassos N, Raptis D, Lell M, et al. Intra-abdominal localized hyaline-vascular Castleman disease: imaging characteristics and management of a rare condition. Arch Med Sci. 2016;12:227-232.

5. Bonekamp D, Horton KM, Hruban RH, Fishman EK. Castleman disease: the great mimic. RadioGraphics. 2011;31:1793-1807.

6. Kim MS, Ju JK, Kim Y. Surgical management of unicentric Castleman's disease in the abdomen. Ann Coloproctol. 2014;30:97-100.

7. Dham A, Peterson BA. Castleman disease. Curr Opin Hematol. 2007;14:354-359.

8. Williams AD, Sanchez A, Hou JS, et al. Retroperitoneal Castleman's disease: advocating a multidisciplinary approach for a rare clinical entity. World Journal of Surgical Oncology. 2014:12:30.

9. Modi P, Trivedi A, Gupta R, Rizvi SJ. Retroperitoneal pararenal Castleman's tumor in an adolescent managed laparoscopically. Journal of Endourology. 2008;22:2451-2454.

10. Chan KL, Lade S, Prince HM, Harrison SJ. Update and new approaches in the treatment of Castleman disease. Journal of Blood Medicine. 2016;7:145-158 